Original Research Article

\title{
Analysis of drug related electrolyte disturbances in emergency medicine department
}

\author{
Karan B. Shah ${ }^{1}$, Sapna D. Gupta ${ }^{2 *}$, Devang A. Rana ${ }^{1}$, Supriya D. Malhotra ${ }^{1}$, Pankaj R. Patel ${ }^{3}$
}

${ }^{1}$ Department of Pharmacology, ${ }^{2}$ Department of Emergency Medicine, ${ }^{3}$ Dean, Smt. NHL Municipal Medical College, Ahmedabad, Gujarat, India

Received: 27 July 2018 Accepted: 21 August 2018

*Correspondence to: Dr. Sapna D. Gupta, Email: sapna_gupta76@ yahoo.com

Copyright: (C) the author(s), publisher and licensee Medip Academy. This is an openaccess article distributed under the terms of the Creative Commons Attribution NonCommercial License, which permits unrestricted noncommercial use, distribution, and reproduction in any medium, provided the original work is properly cited.

\begin{abstract}
Background: Electrolytes play an important role in various physiological functions of the body. Electrolyte disturbances are one of the most common problems encountered in critically ill patients. Drugs are also known to cause adverse electrolyte consequences. These drugs could be anti-hypertensive agents, hormones, antipsychotics or steroids. There is paucity of published literature on electrolyte disturbances caused by drugs. The purpose of our study was to evaluate the electrolyte disturbances caused by various drugs in critically ill patients.

Methods: Following approval of the Institutional Ethics Committee, data collection was started. Adverse Drug Reactions (ADRs) presenting as an electrolyte disturbance in emergency medicine department or occurring in hospitalized patients in the Intensive care unit (ICU) of our hospital was be collected. ADRs resulting into electrolyte disturbances were identified and analysed in detail for demographic details, types of electrolyte disturbances, seriousness, severity, causality and preventability of ADRs. Fisher's exact test was done to find out the statistical difference between the electrolyte disturbances and different drugs.

Results: Total 58 ADRs were reported as an electrolyte disturbance. Mean age of the patients affected was 52.48 years. Highest number of ADRs were observed in the age group of 61 to 70 years. Hypokalemia constituted 32 cases $(55.2 \%)$ followed by hyponatremia $(25.9 \%)$, hyperkalemia $(6.9 \%)$, hypernatremia $(6.9 \%)$, hypocalcemia (1.7\%), hypomagnesemia (1.7\%) and hypophosphatemia $(1.7 \%)$. Insulin was associated with maximum cases of ADRs (27.6\%).

Conclusions: Electrolyte disturbances constitutes a major chunk of ADRs especially in critically ill patients. The physicians must be well-versed with the dynamics of fluid-electrolyte balance.
\end{abstract}

Keywords: Adverse drug reactions, Electrolyte disturbances, Intensive care unit

\section{INTRODUCTION}

Electrolytes play an important role in various physiological functions of the body. It also plays vital role in the functioning of cells, in maintaining tissue perfusion and acid-base balance. Regulation of extra and intracellular concentrations of various electrolytes is crucial for many metabolic processes and organ functions. Kidneys play an important part in maintenance of the levels of electrolytes. ${ }^{1}$ Besides the kidneys, the other mechanisms which are involved in the regulation of fluid and electrolyte balance in the organism are hormones like antidiuretic hormone, aldosterone, and parathyroid hormone and various other factors such as physiological stress and age also play important roles in the regulation of electrolyte balance.

Electrolyte disturbances are one of the most common problems encountered in critically ill patients. ${ }^{2}$ Drugs are also known to cause adverse electrolyte consequences. These drugs could be anti-hypertensive agents like diuretics and drugs affecting the Renin Angiotensin System (ACEIs and ARBs), hormones like insulin, various 
antipsychotics or steroids. Diuretics cause hyponatremia via impairment of urinary diluting capacity. ACEIs and ARBs cause Interference with conversion of angiotensin I into angiotensin II and induces state of hypoaldosteronism leading to hyperkalemia. Antipsychotics produces syndrome of inappropriate antidiuretic hormone secretion (SIADH) which causes hyponatremia. Hypokalemia by insulin is due to stimulation of $\mathrm{Na}^{+} / \mathrm{K}^{+}$ATPase pump. Various steroids enhance sodium reabsorption at the renal distal tubule which ultimately results in hypokalemia.

Hyponatremia is the most frequent electrolyte disturbance accounting for almost $30-40 \%$ of hospitalized patients. ${ }^{3}$ Other common electrolyte disturbances include those of potassium, calcium, phosphorous and magnesium. Incidence of drug related electrolyte disturbances remains largely unknown. ${ }^{4}$ Most critically ill patients have several comorbid disease states, both acute and chronic disease, and are prescribed numerous drugs, all of which may contribute to electrolyte disturbances. ${ }^{4}$

It has been established that the prevalence of electrolyte abnormalities is directly related to mortality and increase in hospitalization period. ${ }^{5}$

There is paucity of published literature on electrolyte disturbances caused by drugs. The purpose of the present study was to evaluate the electrolyte disturbances caused by various drugs in critically ill patients.

The objective of the present study was to analyse of adverse drug reactions (ADRs) presenting as electrolyte disturbances in emergency medicine department

\section{METHODS}

This prospective cross-sectional study was a collaboration of the Department of Pharmacology and the Emergency Medicine Department at Smt. N.H.L Municipal Medical College and V.S General Hospital, Ahmedabad, India and carried over for the period of 12 months from January 2017 to December 2017.

Following approval of the Institutional Ethics Committee, data collection was started. ADRs resulting into electrolyte disturbances were identified and analysed in detail.

\section{Data analysis}

Age and gender were the demographic details of the patient taken in the study.

\section{Type of electrolyte disturbances}

Hyponatremia, hypernatremia, hypokalemia, hyperkalemia, hypomagnesemia, hypocalcemia and hypophosphatemia were taken into consideration. All the disturbances were considered as per the laboratory reference values.

\section{Seriousness of ADRs}

An ADR was assessed as serious if it fulfilled the WHO UMC criteria for a serious adverse drug reaction, which included lethal, life-threatening, permanently disabling, lead to hospital admission, prolongation of hospital stays or classified as an "important medical event".

\section{Severity of ADRs}

Severity of the ADRs was assessed on the basis of modified Hartwig and Siegel criteria (Table 1). These criteria consist of seven severity levels. Level 1 and 2 are considered as mild, 3 and 4 as moderate while 5, 6 and 7 as severe. ${ }^{6}$

\section{Causality assessment of an $A D R$}

Causality of the ADRs was assessed on the basis of WHO causality assessment scale. This classifies causality as certain, probable/likely, possible, unlikely, conditional/unclassified and unassessable/unclassifiable.

\section{Preventability of ADRs}

Preventability of the ADRs were assessed on the basis of Schumock and Thornton criteria (Table 2). These criteria consist of 7 questions and if the answer of any of the question is "yes" then the ADR is preventable."

\section{Statistical analysis}

Statistical analysis was done using Microsoft excel 2013 and IBM SPSS $®$ (Statistical Package for the Social Sciences) version 23. Fisher's exact test was done to find out the statistical difference between the electrolyte disturbances and different drugs.

\section{Reporting ADRs to the authority}

All the ADRs were reported to the nearest PvPI (Pharmacovigilance Programme of India) ADR Monitoring Centre and were submitted to the WHO UMC via VigiFlow.

\section{RESULTS}

During the study period, total 238 ADRs were collected from various departments of VS General Hospital which is attached with Smt. NHL Municipal Medical College, Ahmedabad. Out of the total ADRs, 58 were reported as an electrolyte disturbance. This constitutes almost $1 / 4^{\text {th }}$ (24.36\%) of the total collected ADRs. Mean age of the patients affected was 52.48 years. Out of the total 58 cases, $26(44.8 \%)$ were males and $32(55.2 \%)$ were females. Highest number of ADRs were observed in the age group of 61 to 70 years. All the ADRs were of Type A (Augmented) according to Willis and Brown classification system. In electrolyte disturbances, hypokalemia constituted 32 cases $(55.2 \%)$ followed by hyponatremia 
(15 cases, 25.9\%), hyperkalemia (4 cases, 6.9\%), hypernatremia (4 cases, 6.9\%), hypocalcemia (1 case, $1.7 \%)$, hypomagnesemia (1 case, 1.7\%) and hypophosphatemia (1 case, 1.7\%) (Figure 1).

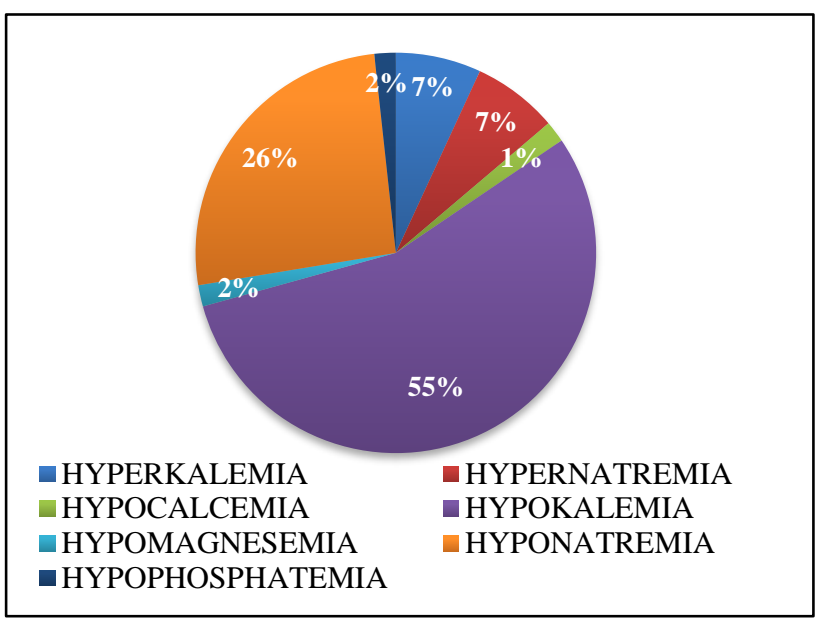

Figure 1: Types of electrolyte disturbances.
As far as the diseases were concerned, 12 patients were diagnosed with hypertension, 16 with diabetes (11 patients with diabetic ketoacidosis), 3 with sepsis and infection each, 2 for subarachnoid hemorrhage and gullian barre syndrome each.

One case the hypokalemia was due to lactulose and methylprednisolone (possible drug interaction). Another case of hyponatremia was possibly due to interaction between torsemide and losartan. Hypokalemia in the patients of diabetic ketoacidosis was possibly due to drug disease interaction with insulin. With respect to hypokalemia, insulin was responsible for maximum number of ADRs and that was statistically significant ( $\mathrm{p}=0.0001$ ) (Figure 2). Considering the individual drugs, insulin was associated with maximum cases of ADRs (27.6\%) followed by furosemide (13.8\%), losartan (6.9\%) and others (Figure 3). Among the total, 30 cases were serious and 28 were non-serious. Causality assessment for 24 ADRs was possible and for remaining 34 ADRs was probable in nature based on standard WHO assessment scale.

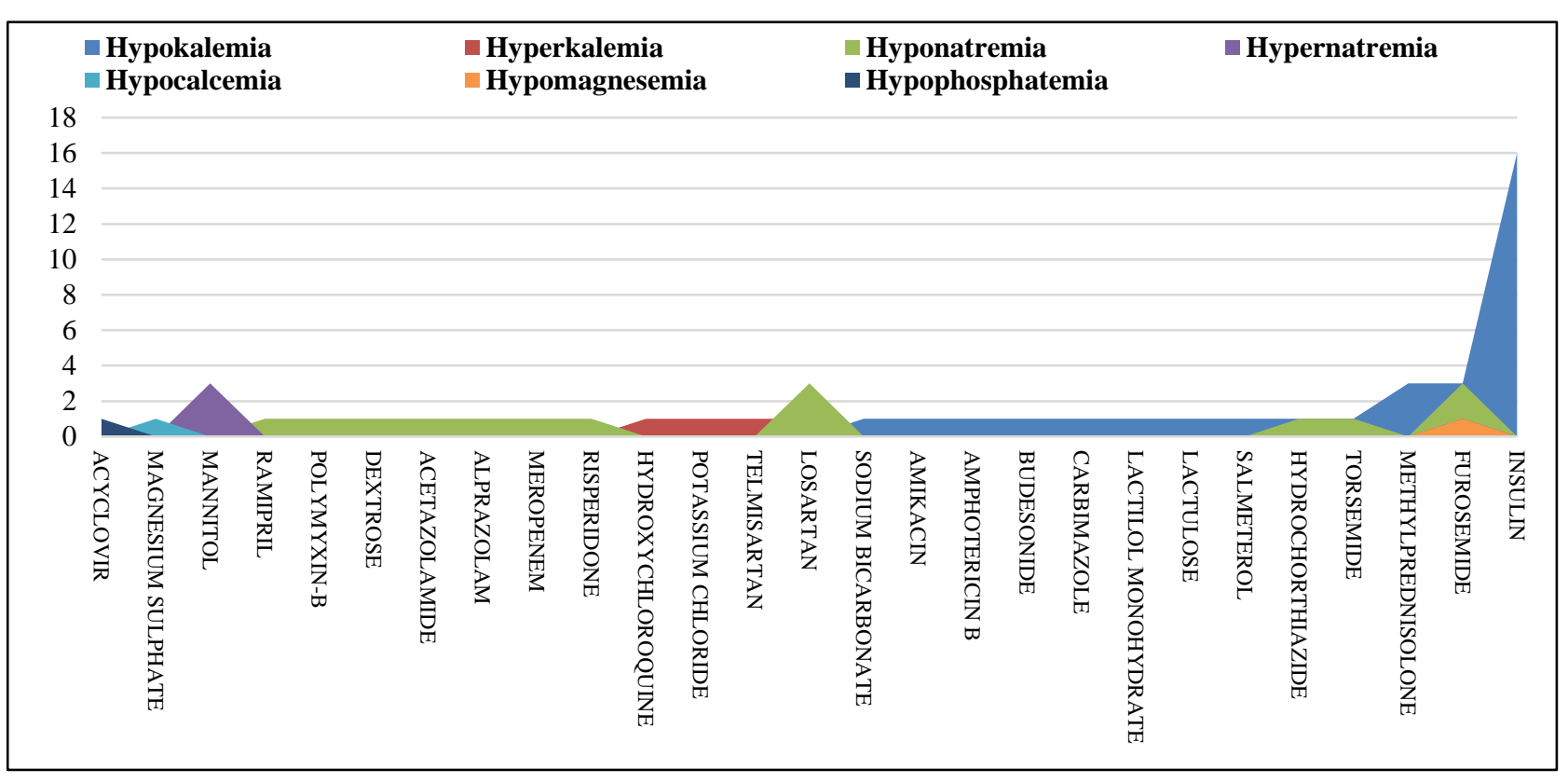

Figure 2: Drugs and types of electrolyte disturbances.

Table 1: Criteria for determining preventability of an ADR.

Answering "yes" to one or more of the following implies that an ADR is preventable.

Was the drug involved inappropriate for the patient's clinical condition?

Was the dose, route, or frequency of administration inappropriate for the patient's age, weight, or disease state?

Was required therapeutic drug monitoring or other necessary laboratory tests not performed?

Was there a history of allergy or previous reactions to the drug?

Was a drug interaction involved in the ADR?

Was a toxic serum drug concentration (or laboratory monitoring test) documented?

Was poor compliance involved in the ADR?

$\mathrm{ADR}=$ adverse drug reaction. 
Table 2: ADR severity assessment scale.

\begin{tabular}{|c|c|}
\hline Levels & Criteria \\
\hline Level 1 & An ADR occurs but requires no change in treatment with the suspected drug. \\
\hline Level 2 & $\begin{array}{l}\text { The ADR requires that the suspected drug be withheld, discontinued, or otherwise changed. No antidote } \\
\text { or other treatment is required, and there is no increase in length of stay. }\end{array}$ \\
\hline Level 3 & $\begin{array}{l}\text { The ADR requires that the suspected drug be withheld, dis- continued, or otherwise changed, and/or an } \\
\text { antidote or other treatment is required. There is no increase in length of stay. }\end{array}$ \\
\hline \multirow{2}{*}{ Level 4} & (a) Any level 3 ADR that increases length of stay by at least one day, or \\
\hline & (b) The ADR is the reason for admission. \\
\hline Level 5 & Any level 4 ADR that requires intensive medical care. \\
\hline Level 6 & The adverse reaction causes permanent harm to the patient. \\
\hline Level 7 & The adverse reaction either directly or indirectly leads to the death of the patient. \\
\hline
\end{tabular}

Non-preventable ADRs were 37 in number and 21 were preventable. 29 ADRs were of level 3, 25 of level 2 and 4 of level 4 severity. About 26 patients were having recovering outcome followed by 19 and 13 for recovered and unknown outcome respectively (Table 3 ).

Table 3: Preventable and nonpreventable ADRs categorized by patient and ADR variables.

\begin{tabular}{|c|c|c|}
\hline Variable & $\begin{array}{l}\text { Preventable } \\
(\%)\end{array}$ & $\begin{array}{l}\text { Non- } \\
\text { preventable }\end{array}$ \\
\hline \multicolumn{3}{|l|}{ Age } \\
\hline $11-20$ & 5 & 2 \\
\hline $21-30$ & 0 & 4 \\
\hline $31-40$ & 0 & 4 \\
\hline $41-50$ & 2 & 6 \\
\hline $51-60$ & 3 & 6 \\
\hline $61-70$ & 7 & 8 \\
\hline $71-80$ & 3 & 5 \\
\hline $81-90$ & 1 & 1 \\
\hline $91-100$ & 0 & 1 \\
\hline \multicolumn{3}{|l|}{ Gender } \\
\hline Male & 10 & 16 \\
\hline Female & 11 & 21 \\
\hline \multicolumn{3}{|l|}{ Severity } \\
\hline Mild & 5 & 20 \\
\hline Moderate & 16 & 17 \\
\hline Severe & 0 & 0 \\
\hline \multicolumn{3}{|l|}{ Seriousness } \\
\hline Serious & 12 & 18 \\
\hline Non serious & 9 & 19 \\
\hline \multicolumn{3}{|c|}{ Electrolyte disturbance } \\
\hline Hypokalemia & 16 & 16 \\
\hline Hyperkalemia & 1 & 3 \\
\hline Hyponatremia & 4 & 11 \\
\hline Hypernatremia & 0 & 4 \\
\hline Hypocalcemia & 0 & 1 \\
\hline Hypomagnesemia & 0 & 1 \\
\hline Hypophosphatemia & 0 & 1 \\
\hline
\end{tabular}

\section{DISCUSSION}

Mean age of patients in present study was 52.48 years. Elderly patients were affected the most with highest numbers of patients being 61 to 70 years of age (15, $25.86 \%$ ). The elderly patients are particularly at increased risk of adverse drug reactions (ADR) due to polypharmacy and physiological changes affecting the pharmacokinetics and pharmacodynamics of many drugs or poor compliance due to cognitive impairment or depression. ${ }^{8}$ There was a female preponderance $32(55.2 \%)$ in present study. This is in contrast with other study done by Khorasani et al, in which there was a male preponderance. The same study had hyponatremia as the most common electrolyte disturbance which is in contrast to present study which is having hypokalemia $(55.2 \%)$ as the most common disturbance followed by hyponatremia $(25.9 \%) .{ }^{5}$ Majority of hypokalemia $(50 \%)$ was due to insulin followed by loop diuretics and glucocorticoids (Figure 3).

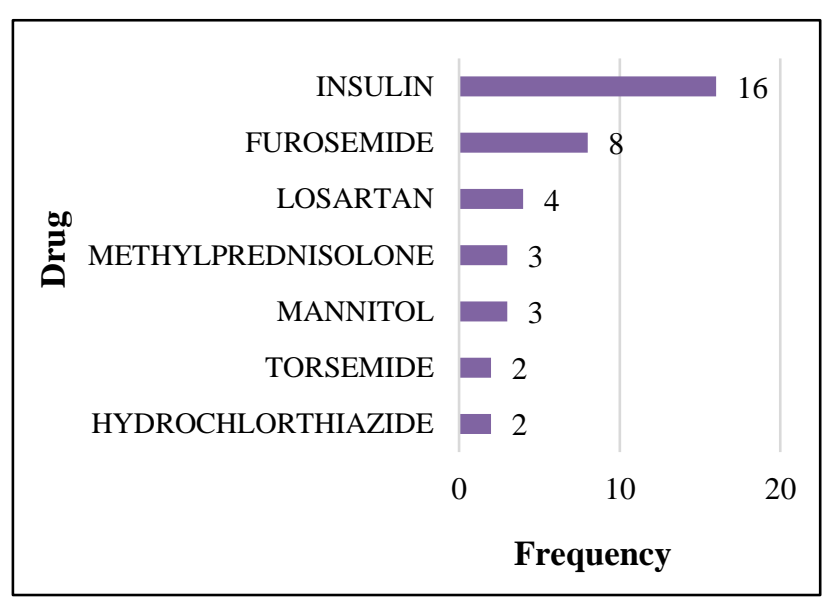

Figure 3: Common drugs

Exogenous insulin can induce mild hypokalemia because it promotes the entry of $\mathrm{K}^{+}$into skeletal muscles and hepatic cells by increasing the activity of the $\mathrm{Na}^{+}-\mathrm{K}^{+}$- ATPase pump. ${ }^{9}$ Increase in epinephrine secretion due to insulin induced hypoglycemia may also contribute. ${ }^{10}$ 
The major setting in which insulin administration leads to hypokalemia is during the treatment of diabetic ketoacidosis as the majority of patients with diabetic ketoacidosis (DKA) and HHS are markedly $\mathrm{K}^{+}$-depleted. ${ }^{11}$ Female preponderance was seen as far as hypokalemia is concerned $(19,59.37 \%)$ which was similar to the study done by Khorasani et al. ${ }^{5}$

Majority of ADRs were severe (33, 56.89\%). Majority of ADRs were non-preventable in nature (37, 63.79\%). Causality assessment of most of the ADRs were probable $(34,58.62 \%)$ followed by possible $(24,41.38 \%)$. Maximum patients were suffering from diabetes mellitus $(16,27.58 \%)$ followed by hypertension, sepsis, infection and others. More than half of the ADRs were serious (30, $51.72 \%)$. As far as the outcome of the ADR is concerned majority of patients were recovering or recovered from the ADR (45, 77.58\%).

Metabolic disturbances come under the heading of predictable ADRs. ${ }^{12}$ Thus, all the ADRs were predictable in nature. Various drug-drug or drug disease interactions can also cause electrolyte disturbances. When diuretics are given along with angiotensin converting enzyme inhibitors (ACEIs) hyponatremia can occur. Hypokalemia may aggravate in the patients of Diabetic ketoacidosis when they are given insulin. Almost half of the patients who developed hyponatremia was suffering from hypertension and were on either ACEIs, ARBs or diuretics.

There are also some limitations of the study like only the critically ill patients (patients admitted under emergency medicine department) were taken and so it may not actually reveal the real prevalence of electrolyte disturbances in general patient population.

\section{CONCLUSION}

Hence in the present study authors observed that electrolyte disturbances constitute a major chunk of ADRs especially in critically ill patients. Studies concentrating on drug induced electrolyte disturbances are few, if any. Present study is one of its kind focusing on the said issue. Hence, a larger study including all the patient population should be conducted with special focus on elderly population. Because of the high incidence rate of electrolyte abnormalities, the physicians must be well-versed with the dynamics of fluid-electrolyte balance.

So, through timely identification, a thorough knowledge and understanding of the general electrolyte disorders and drugs causing them will ensure their prevention and appropriate treatment if needed.
Funding: No funding sources

Conflict of interest: None declared

Ethical approval: The study was approved by the Institutional Ethics Committee

\section{REFERENCES}

1. Bockenkamp B, Vyas $\mathrm{H}$. Understanding and managing acute fluid and electrolyte disturbances. Current Paediatr. 2003;13(7):520-8.

2. Kraft MD, Btaiche IF, Sacks GS, Kudsk KA. Treatment of electrolyte disorders in adult patients in the intensive care unit. Am J Health System Pharma. 2005 Aug 15;62(16):1663-82.

3. Patterson J. The Impact of Hyponatremia. Pharmacotherapy. 2011;31(5S):5S-8S.

4. Buckley MS, LeBlanc JM, Cawley MJ. Electrolyte disturbances associated with commonly prescribed medications in the intensive care unit. Critical Care Medicine. 2010 Jun 1;38:S253-64.

5. Khorasani B, Khorasani AG. Evaluating mortality rate caused by electrolyte abnormalities in patients hospitalized. Acta Medica Iranica. 2008;46(2):141-8.

6. Schumock GT, Thornton JP. Focusing on the preventability of adverse drug reactions. Hospital Pharma. 1992 Jun;27(6):538.

7. Hartwig SC, Siegel J, Schneider PJ. Preventability and severity assessment in reporting adverse drug reactions. Am J Health System Pharma. 1992 Sep 1;49(9):2229-32

8. Tangiisuran B, Wright $\mathrm{J}$, Van der Cammen $\mathrm{T}$, Rajkumar C. Adverse drug reactions in elderly: challenges in identification and improving preventative strategies. Age Ageing. 2009;38(4):358359.

9. Minaker K, Rowe J. Potassium homeostasis during hyperinsulinemia: effect of insulin level, betablockade, and age. Am J Physiol. 1982;242(6):373-7.

10. Petersen K, Schluter K, Kerp L. Regulation of serum potassium during insulin-induced hypoglycemia. Diabetes. 1982;31(7):615-7.

11. Liamis G, Liberopoulos E, Barkas F, Elisaf M. Diabetes mellitus and electrolyte disorders. World J Clin Cases 2014;2(10):488-96.

12. Breathnach SM, Hintner H. Classification and mechanisms of drugs reactions. Adverse Drug Reactions Skin. 1992:14-38.

Cite this article as: Shah KB, Gupta SD, Rana DA, Malhotra SD. Patel PR. Analysis of drug related electrolyte disturbances in emergency medicine department. Int J Basic Clin Pharmacol 2018;7:20059. 